\title{
Integrating driving and traffic simulators for the study of railway level crossing safety interventions: a methodology
}

\author{
G. S. Larue ${ }^{1}$, I. Kim² ${ }^{2}$, A. Rakotonirainy ${ }^{1}$, L. Ferreira ${ }^{2}$ \\ \& N. Haworth ${ }^{1}$ \\ ${ }^{1}$ Centre for Accident Research and Road Safety - Queensland, \\ Queensland University of Technology, Brisbane, Australia \\ ${ }^{2}$ Faculty of Engineering, Architecture and Information Technology, \\ The University of Queensland, Brisbane, Australia
}

\begin{abstract}
Safety at Railway Level Crossings (RLXs) is an important issue within the Australian transport system. Crashes at RLXs involving road vehicles in Australia are estimated to cost $\$ 10$ million each year. Such crashes are mainly due to human factors; unintentional errors contribute to $46 \%$ of all fatal collisions and are far more common than deliberate violations. This suggests that innovative intervention targeting drivers is particularly promising to help improve RLX safety. In recent years there has been a rapid development of a variety of affordable technologies which can be used to increase driver's risk awareness around crossings. To date, no research has evaluated the potential effects of such technologies at RLXs in terms of safety, traffic and acceptance of the technology. Integrating driving and traffic simulations is a safe and affordable approach for evaluating these effects. This methodology will be implemented in a driving simulator, where we recreated realistic driving scenario with typical road environments and realistic traffic. This paper presents a methodology for evaluating comprehensively potential benefits and negative effects of such interventions: this methodology evaluates driver awareness at RLXs, driver distraction and workload when using the technology. Subjective assessment on perceived usefulness and ease of use of the technology is obtained from standard questionnaires. Driving simulation will provide a model of driving behaviour at RLXs which will be used to estimate the effects of such new technology on a road
\end{abstract}


network featuring RLX for different market penetrations using a traffic simulation. This methodology can assist in evaluating future safety interventions at RLXs. Keywords: railway level crossings, intelligent transport systems, driving and traffic simulations

\section{Introduction}

Crash data are traditionally gathered through police records. Safety assessments of traffic systems are usually analysed based on particular police-reported historical crash data and are therefore reactive. However, it is unreasonable to wait for a large enough number of crashes to occur before traffic systems can be evaluated especially at RLX where police-reported historical records may not be sufficient (since collisions at RLX are rare but catastrophic) for analysis either quantitatively or qualitatively.

There is consistent evidence that the behaviour of drivers contributes to crashes and near miss incidents at Railway Level Crossings (RLXs) [1,2]. The development of innovative approaches which directly target driver behaviour is a highly promising approach to improve RLX safety. Indeed, in recent years there has been a rapid growth in the development of a variety of emerging technologies [3]. A review of the literature shows that safety at both passive and active RLXs could be improved by Intelligent Transport Systems (ITS) interventions, and that ITS should focus on providing drivers with simple, easy to process information about the approach of trains. Queensland drivers recruited into earlier focus groups perceived ITS interventions as potentially useful. However, this positive perception primarily related to their role in alerting the driver that a train is approaching, especially in situations where visibility is reduced.

New technologies have the potential to increase driver awareness at RLXs, using either in-vehicle or on-road warnings. However, it is important to assess whether driver behaviour will really improve during a driving task with the technology, whether new messages will not generate negative effects on their attention on the road and whether drivers would accept the new information provided to them. It is also important to asses the effects of such interventions on traffic around crossings. Indeed, increased queuing issues around crossings have been shown to result in increased risky behaviours. No study has focused on such issues, and this paper proposes a methodology for evaluating new ITS interventions for RLXs. Such assessment requires to obtain information about driver's behaviour when using the technology as well as information about effects on traffic when implemented within a road network. Therefore, integrating diving and traffic simulations is the solution to obtain a comprehensive, safe and cost-effective evaluation of potential ITS interventions for RLXs.

This paper presents a methodology which provides a comprehensive assessment of driver behaviour at RLXs and allows an assessment of the benefits and disadvantages of a new ITS intervention. A wide range of measures from the driver, the vehicle and the environment will be compared between a baseline of traditional RLXs and RLXs equipped with new ITS interventions. These measures provide a 
comprehensive view of the effects of a new ITS intervention as they cover driver awareness at the crossing and safety of the driving behaviour, driver distraction, driver workload, driver acceptance of the technology and potential negative effects on traffic. Each dimension of the analysis of the effects of an ITS intervention although likely to be interdependent - will be the focus of a section in this paper.

\section{Driver's situation awareness at RLXs}

In general, good drivers are more aware of their surroundings. This ability is embedded in the construct of Situation Awareness (SA), which is defined as "the perception of the elements in the environment within a volume of time and space, the comprehension of their meaning, and the projection of their status in the near future" [4].

The best way to measure SA is to use freeze-probe techniques [4]. Nevertheless, such techniques are criticised for their intrusion upon primary task performance [5], which is an issue when trying to evaluate the driving behaviour around RLXs. SA can also be assessed indirectly though behavioural and performance measures such as the time required to respond and the accuracy of the response to an event [6], or process indices such as the measurement of participant eye movements to determine how the participant's attention was allocated [5]. Such methods have limitations: changes in performance are not always related to changes in SA and eye movements might reflect where the driver looks rather than what the driver sees [4]. Nevertheless positive associations between SA and driving performance (lane positioning, headway distance) have been found during a simulated driving task $[6,7]$ and indirect methods are preferred for this methodology.

As applied to railway crossings, performance and errors (related to the capacity to project the future status of elements in the driving environment) can be obtained by an analysis of the compliance rate and violation of the crossings, an assessment of the safety of the speed when arriving at the crossing and an assessment of reaction times when an active protection is activated or when a static sign is legible.

\subsection{Performance measures}

\subsection{Compliance rate/violation at the crossing}

Compliance rate at railway level crossings is defined as the complete adherence to the traffic rules at the crossing. It is a measure that is widely used to assess the safety of a railway level crossing. It has been shown that compliance rates obtained on a driving simulator are consistent with observations made on roads [8].

A driver's approach to a crossing is classified as "safe" if, at the distance to the RLX where they first look for information, it is possible to (i) stop the vehicle before the crossing and (ii) proceed ahead of a hypothetical train approaching from the furthest observable point on the track [9].

The position where the driver starts their final braking is another measure of interest for evaluating the safety of the approach of the RLX. This measure directly 
contributes to the collision/near-miss likelihood, as the later the braking, the shorter the time-to-collision, and hence the higher the possibility of a collision [8].

The speed profile while approaching a crossing is also of interest. Driving at faster speeds reduces the time a driver has to gather and process information from their environment, to formulate and carry out an action in order to respond safely to a critical event [10]. The speed profile provides information about the value of speed, the time and distance to the crossing when speed starts to decrease and the way speed is reduced (abruptly, smoothly), and hence provides information about the safety of the approach. The braking force can also be investigated as it is an accepted method to investigate the urgency with which the driver reacts to a situation [10].

\subsection{Process indices}

\subsection{Safety of driver's approach of railway crossings}

The driver's evaluation of the situation can be monitored through their eye gaze patterns. Driver gazes at RLXs can explain driver errors related to detecting the crossing or the approach of a train. Eye movement analyses have shown that most drivers do not look at several signs when approaching RLXs. Further to the lack of gazes at signs when approaching crossings, a large number of drivers do not search for trains at passive crossings [11].

\section{Driver's attention toward the road}

The use of HMIs designed to assist in various driving situations is increasing (e.g., collision warning and avoidance, adaptive cruise control, lane departure, etc.). Although these systems are designed to benefit drivers, there are many instances where they can, and do, distract drivers from the primary task of driving. This is primarily due to the complexity involved, on the driver's behalf, in setting and monitoring the interface whilst driving. Therefore the effects of any new ITS interventions on driver distraction should be investigated. Such investigation will be done by analysing gaze patterns of the driver - which will provide information about their gaze fixations on signs at various crossings as well as the of their gaze distraction from the road. The driver's control of the vehicle will also be monitored, through lane keeping for instance.

\subsection{Reaction times}

The braking reaction time (BRT) is of interest for RLXs. BRT is defined as the time elapsed from the moment a stimulus appears until the driver presses the brake pedal [12]. BRT is considered safe if braking occurs within 2 seconds after the onset of a stimulus when driving at highway speeds (around $80 \mathrm{kph}$ ) [13]. It is of interest to assess whether the different ITS interventions improve BRT when compared to conventional crossings. It is expected that BRT should be faster with 
the assistance of ITS. Nevertheless, if the ITS intervention increases driver mental workload, it could reduce their attention and increase their BRT.

\subsection{Control of the vehicle}

The common perception among road safety researchers is that, if a manipulation causes a driver to deviate more within the lane, and especially if the vehicle is seen to cross one or both of the lane boundaries, then the risk of a crash is also increased [10]. Performing a secondary task while driving - such as the using an ITS - has been shown to increase lane position variability in both simulated and on-road driving environments $[14,15]$. Therefore, lateral vehicle behaviour - or lane positioning - is an important indicator to evaluate in order to assess whether the ITS intervention distracts the driver from the driving task.

\subsection{Eye gaze patterns}

The time spent on searching the information should be long enough to be aware of the situation, but should not be too long in order to be able to react to other events. Glances at signs for more than two seconds would be considered as visually too distracting [16]. Therefore, we can similarly evaluate whether an ITS intervention takes driver's attention away from the road for more than 2 seconds. Also, if the task becomes too demanding for the driver, their visual field concentrates towards the road centre and results in less time looking at areas in the periphery $[14,17]$.

\section{Driver workload}

In the driving context, workload is commonly defined as the effort required to maintain the driving state within a subjective safety zone [18]. There are four methods which can be used to measure driver workload [19]: (i) secondary task performance, (ii) subjective estimates of workload, (iii) physiological measures and (iv) stand-alone performance measures.

While secondary task performance is one of the most commonly used measures of workload in driving research, is not an appropriate solution in this study, the focus being on assessing effects of a new ITS intervention during a realistic driving task.

Subjective estimates of driver workload are usually comprised of one or more questions presented in a questionnaire format which are designed to probe a driver's experience of workload. The most commonly used and the most widely validated subjective workload questionnaire is the NASA Task Load Index, or NASA-TLX [20]. The NASA-TLX is a multidimensional rating instrument that assesses six dimensions of subjective workload: mental demand, physical demand, temporal demand, performance, effort, and frustration level.

Physiological measures are also used in driving research to assess mental workload. For example, cardiac activity has been found to be related to the amount of workload experienced by a subject [21]. Most studies show that the 
metric heart rate increases and the metric heart rate variability decreases during increased mental processing [22, 23], though contradictory results have been found. In addition, indices derived from spectral analysis of inter-beat-interval (IBI) variability have been related to mental effort, highlighting that attention is required to carry out a task [24].

An example of a stand-alone performance measure for RLX intervention is driver eye glance behaviour. In particular, increased glance duration and greater frequency of glances to a particular area in a driver's visual field. These metrics are generally accepted as measures of increased visual workload [19].

Three out of the four methods will be implemented in order to get a comprehensive evaluation of workload with the different ITS interventions. Eye gaze patterns and cardiac activity will be measured when arriving at RLXs. Effects of the ITS will be compared to a baseline of traditional RLXs. Participants will also provide subjective assessment by answering the NASA-TLX at the end of their driving session.

\section{Acceptance of the technology}

Current emerging technologies are likely to improve detection of crossings and trains, but they largely fail to address the need to eliminate the ability of the driver to circumvent the technology. To ensure compliance, it is necessary there are high levels of driver acceptability of RLX warning devices, such that motorists perceive the system to be reliable and understand the need for the device [25]. ITS interventions will be efficient in reducing crashes at RLXs only if such technologies are deemed to be acceptable by drivers. That is why it is important to assess whether new ITS interventions are likely to be accepted by drivers.

User acceptance - as defined by Swanson [26] - is a "potential user's predisposition toward personally using a specific system". Research supports a strong causal relationship between the behavioural intention to use a new technology and the actual behaviour [27]. The technology acceptance model (TAM) is a useful model for predicting intention to use or actual use of a new technology. Nevertheless, it needs to be integrated into a broader model including variables related to both human and social factors, since most behaviours depend at least to some degree on non-motivational factors (such as time, money, skills, cooperation) and are not completely under volitional control. TPB takes these factors into account and more and more studies have been focused on integrating them [28]. The TAM is an adaptation of the theory of reasoned action (TRA) which explains and predicts the behaviour of people in specific situations [29]. The assumption in the TAM is that the behaviour is volitional, that is to say using the technology is voluntary or at the discretion of the user [30]. It is primarily used to predict the likelihood of adoption of a new technology. TPB differs from the TRA by adding the construct perceived behavioural control, which refers to people's perception of the ease or difficulty of performing the behaviour of interest. Such construct varies across situations and actions, and this model is widely researched and used for predicting and explaining human behaviour across 
a variety of conditions, while also considering the effects of individual and social systems in the process [31].

TAM focuses on two user beliefs, namely the perceived ease of use and the perceived usefulness of the new technology, and their influences on the intention to adopt a new technology [32]. Even if potential users perceive a given technology to be useful, they may also believe that the system is too hard to use and that the performance benefits of usage are outweighed by the effort of using the technology [33].

The TAM was proposed as a means of predicting technology usage. However, most studies in literature validate it by using a measure of behavioural intention to use or self-reported usage, rather than actual usage. TAM variables are a much stronger predictor of the behavioural intention to use a technology than the actual usage of a technology [34]. Such measures are therefore not precise measures and should be used as relative indicators [29]. When users have experiences in using the technology, the relationships between intention and self-report and actual usage are stronger. When using a pre-implementation perspective (the case of this study), it is necessary to have an experimental design in which the subjects were introduced and trained to use a new technology before they provide responses regarding the perceived usefulness, perceived ease of use and behavioural intentions toward that particular technology [32].

TPB identifies three attitudinal antecedents of behavioural intention. Two reflect the perceived desirability of performing the behaviour and are (i) the attitude toward outcomes of the behaviour (reflects positive feelings towards performing a particular behaviour) and (ii) the subjective norm (reflects perceptions of social pressure to perform in a particular way). The third construct is the perceived behavioural control, which measures and accounts explicitly for the extent to which users perceive that they have control over their behaviour [30,31], i.e. it reflects internal and external constraints on the ability to perform the action [28].

In the Theory of Planned Behaviour, behavioural control directly affects intention to perform a behaviour, and may directly affect behaviour in situations where the user intends to perform the behaviour, but is prevented from doing so [31].

\section{Effects of the technology on traffic}

Lee \& $\mathrm{Hu}$ [35] emphasized that delay was one of the prime indications for evaluating RLX as they might create excessive delay for road traffic and therefore possible violations. Therefore effects on traffic around RLXs of new ITS interventions need to be investigated. An innovative traffic conflict prediction model and a queuing effect model using current transportation simulation are developed for such an assessment. This model estimates the safety of railway crossings as a function of the traffic volume, the train headway and the type and market penetration of the trialled technology.

The idea of using traffic simulation for safety assessment usually faces some difficulties due to the fact that all road users drive a vehicle in a safe manner. 
In order to overcome this issue, extensions to the traffic simulator need to be developed (through the API of the traffic simulator). Vehicles dynamics around crossings with ITS interventions need to be added to the traffic simulation. This is done by using the data collected from the driving simulator as inputs to the traffic simulation model. This provides a range of driver perception, reaction and driving behaviour and errors, which can result in potential conflicts at RLXs.

\subsection{Traffic conflicts}

Compliance can be interpreted as the probability of stopping in a traffic simulator. During the time of activation of a signal (flashing light at RLX for instance), the driver makes a decision to either 'go' or 'stop' depending on their speed and distance to a stop line. This probability can be modelled as a function of speed and distance to the crossing when the signal is activated. The probability model $p$ is given by the following equation:

$$
p=\frac{1}{1+\exp ^{-\left(\alpha+\beta_{1} \cdot v+\beta_{2} \cdot d\right)}}
$$

where $v$ is the speed of the vehicle, $d$ is the distance to the stopping position and $\alpha, \beta_{1}$ and $\beta_{2}$ are constants.

The speed profile observed in the driving simulator can be used to reliably model the speed of the leading vehicle approaching a RLX in the traffic simulation. This is done by a calibration of the speed of the leading vehicle. This calibration changes the values of the coefficients of the previous equation. Using values of coefficients measured in a driving simulator, it is possible to create traffic in the traffic simulation that models adequately a real situation in terms of the probability of stopping. Then potential conflicts at the crossing can be evaluated with microsimulation as well as their severity.

\subsection{Delay and queue length}

Micro-simulation is used to study traffic queues. Such a study can help resolve the likelihood of conflicts and also can help practitioners to analyse traffic flow interactions. Delay and queue length are appropriate measures not only of traffic efficiency but also of traffic safety. Here, the definition of delay is the time gap between the moment when a vehicle stops, as defined by its speed being under a given threshold speed, and the moment when the vehicle's speed exceeds another threshold speed. In particular, when some drivers experience delays near a RLX, they tend to change their driving behaviour and may cross the crossing illegally, make a U-turn to find an alternative route, or experience reduced attention. Such conditions are likely to result in unsafe practices. From several earlier studies, a simple empirical model was provided to estimate the queue length at the intersections [36, 37]. This model can be used to assess whether a particular RLX configuration is likely to be risky. Then such RLX can be implemented on the traffic simulator, and effects of the new ITS interventions can be simulated. 
Clearance time is also a key traffic element for assessing the effects of ITS interventions at RLXs. Clearance time is calculated as a function of the distance between the stopping location at an intersection and the RLX, the speed of the shock wave, and the type of vehicles in the queue.

\section{Conclusion}

This paper has presented a methodology to evaluate new ITS interventions at railway level crossings. This methodology provides a comprehensive evaluation of the potential positive and negative effects of the implementation of new ITS at RLXs. Driver behaviour while approaching a crossing will be measured by a comprehensive set of variables collected in a driving simulator, together with physiological devices. The effects of the technology on driver workload will be monitored with both physiological devices and subjective questionnaires. Driver behaviour will be assessed through compliance at the crossing, the safety of the approach of the crossing, the control of the vehicle, driver's eye movements and fixations on signals, rail track and/or trains, and driver workload (subjective and physiological). After participants have used the ITS, their feedback about their acceptance of the technology will be collected via a questionnaire which is an adaptation of the Technology Acceptance Model. Driving simulator data will then be used to create a realistic micro-traffic simulation in order to evaluate the effects of an ITS intervention on a network scale around a crossing, focusing on both potential conflicts and queuing effects due to the technology.

This project will evaluate driver reactions to the introduction of new ITS interventions at RLXs. The most promising in-vehicle and on-road ITS which are likely to facilitate RLX safety outcomes have been identified during a literature review process and will be evaluated with the use of both a driving and traffic simulators. By integrating surrogate safety measures in conjunction with traffic simulation models, we will be able to determine which safety approach is safer and more efficient for specified traffic and train volumes and market penetration of a new ITS intervention for RLXs. This will result in a ranking of the trialled ITS interventions and recommendations for actual implementations.

\section{Acknowledgement}

The authors gratefully acknowledge the CRC for Rail Innovation (established and supported under the Australian Government's Cooperative Research Centres program) for the funding of this research. Project No. R2.111.

\section{References}

[1] Australian Transport Safety Bureau, Level crossing accidents - fatal crashes at level crossings. Technical report, Australian Transport Safety Bureau, 2002. 
[2] Edquist, J., Stephan, K. \& Wigglesworth, L., A literature review of human factors safety issues at australian level crossings. Technical report, Monash University Accident Research Centre, 2009.

[3] Tey, L.S., Ferreira, L. \& Dia, H., Evaluating cost-effective railway level crossing protection systems, 2009.

[4] Endsley, M.R. \& Jones, D.G., Designing for Situation Awareness: An Approach to User-Centered Design. CRC Press: New York, 2nd edition, 2010.

[5] Salmon, P., Stanton, N., Walker, G. \& Green, D., Situation awareness measurement: A review of applicability for c4i environments. Applied Ergonomics, 37(2), pp. 225-238, 2006.

[6] Ma, R. \& Kaber, D.B., Situation awareness and workload in driving while using adaptive cruise control and a cell phone. International Journal of Industrial Ergonomics, 35(10), pp. 939-953, 2005.

[7] Bolstad, C.A., Situation awareness: Does it change with age? Proceedings of the Human Factors and Ergonomics Society Annual Meeting, 45(4), pp. 272-276, 2001.

[8] Tey, L.S., Ferreira, L. \& Wallace, A., Measuring driver responses at railway level crossings. Accident Analysis \& Prevention, 43(6), pp. 2134-2141, 2011.

[9] Ward, N.J. \& Wilde, G.J.S., Driver approach behaviour at an unprotected railway crossing before and after enhancement of lateral sight distances: An experimental investigation of a risk perception and behavioural compensation hypothesis. Safety Science, 22(1-3), pp. 63-75, 1996.

[10] Rudin-Brown, C.M., Lenne, M.G., Edquist, J., Trotter, M., Navarro, J. \& Tomasevic, N., Driver compliance with, and understanding of, level crossing controls. Australasian Road Safety Research, Policing and Education Conference.

[11] Wigglesworth, E., Human factors in level crossing accidents. Accident Analysis \& Prevention, 10(3), pp. 229-240, 1978.

[12] Shinar, D., Traffic Safety and Human Behavior. Emerald Group Publishing Limited: Burlington, 2007.

[13] Rudin-Brown, C.M. \& Parker, H.A., Behavioural adaptation to adaptive cruise control (acc): implications for preventive strategies. Transportation Research Part F: Traffic Psychology and Behaviour, 7(2), pp. 59-76, 2004.

[14] Engstrom, J., Johansson, E. \& Ostlund, J., Effects of visual and cognitive load in real and simulated motorway driving. Transportation Research Part F: Traffic Psychology and Behaviour, 8(2), pp. 97-120, 2005.

[15] Jamson, H.A. \& Merat, N., Surrogate in-vehicle information systems and driver behaviour: Effects of visual and cognitive load in simulated rural driving. Transportation Research Part F: Traffic Psychology and Behaviour, 8(2), pp. 79-96, 2005.

[16] Rail Safety and Standards Board, Research into traffic signs and signals at level crossings. Technical report, 2011. 
[17] Harbluk, J.L., Noy, Y.I., Trbovich, P.L. \& Eizenman, M., An on-road assessment of cognitive distraction: Impacts on drivers' visual behavior and braking performance. Accident Analysis \& Prevention, 39(2), pp. 372-379, 2007.

[18] Boer, E., Behavioural entropy as a measure of driving performance. third international driving symposium on human factors in driver assessment, training and vehicle design, p. 225-229, 2005.

[19] Gawron, V.J., Human workload, CRC Press: Boca Raton, pp. 87-230, 2008.

[20] Hart, S.G. \& Staveland, L.E., Development of NASA-TLX (Task Load Index): Results of empirical and theoretical research, Elsevier: Amsterdam, Holland, 1987.

[21] Cinaz, B., Arnrich, B., La Marca, R. \& Tröster, G., Monitoring of mental workload levels during an everyday life office-work scenario. Accepted for Personal and Ubiquitous Computing Journal, 2011.

[22] Ahsberg, E., Gamberale, F. \& Gustafsson, K., Perceived fatigue after mental work: An experimental evaluation of a fatigue inventory. Ergonomics, 43(2), pp. 252-268, 2000.

[23] Oron-Gilad, T., Ronen, A. \& Shinar, D., Alertness maintaining tasks (amts) while driving. Accident Analysis \& Prevention, 40(3), pp. 851-860, 2008.

[24] Tejero, P. \& Choliz, M., Driving on the motorway: The effect of alternating speed on driver's activation level and mental effort. Ergonomics, 45(9), pp. 605-618, 2002.

[25] Abraham, J., Datta, T. \& Datta, S., Driver behaviour at rail-highway crossings. Transportation Research Record, 1648, pp. 28-34, 1998.

[26] Swanson, E., Information system implementation: bridging the gap between design and utilization. Irwin, 1988.

[27] Chau, P.Y.K. \& Hu, P.J.H., Information technology acceptance by individual professionals: A model comparison approach. Decision Sciences, 32(4), pp. 699-719, 2001.

[28] Fu, J.R., Farn, C.K. \& Chao, W.P., Acceptance of electronic tax filing: A study of taxpayer intentions. Information \& Management, 43(1), pp. 109126, 2006.

[29] Legris, P., Ingham, J. \& Collerette, P., Why do people use information technology? A critical review of the technology acceptance model. Information \& Management, 40(3), pp. 191-204, 2003.

[30] Dishaw, M.T. \& Strong, D.M., Extending the technology acceptance model with task-technology fit constructs. Information \& Management, 36(1), pp. 9-21, 1999.

[31] Ajzen, I., The theory of planned behavior. Organizational Behavior \& Human Decision Processes, 50(2), p. 179, 1991.

[32] Wu, C.S., Cheng, F.F., Yen, D.C. \& Huang, Y.W., User acceptance of wireless technology in organizations: A comparison of alternative models. Computer Standards \& Interfaces, 33(1), pp. 50-58, 2011.

[33] Davis, F.D., Perceived usefulness, perceived ease of use, and user acceptance of information technology. MIS Quarterly, 13(3), pp. 319-340, 1989. 
[34] Turner, M., Kitchenham, B., Brereton, P., Charters, S. \& Budgen, D., Does the technology acceptance model predict actual use? a systematic literature review. Information and Software Technology, 52(5), pp. 463-479, 2010.

[35] Lee, C.K. \& Hu, S.R., Accident risk at a railway level crossing. Journal of Eastern Asia Society for Transportation Studies, 7, pp. 53-61.

[36] Sayed, T., Brown, G. \& Navin, F., Simulation of traffic conflicts at unsignalised intersections with tsc-sim. Accident Analysis \& Prevention, 26(5), pp. 593-607, 1994.

[37] Archer, J., Traffic conflict technique - historical to current state-of-the-art. Technical report, Institution for Infrastructure, Royal Institute of Technology, 2001. 